\title{
Evaluation of the Ability of Miltefosine Associated with Topical GM-CSF in Modulating the Immune Response of Patients with Cutaneous Leishmaniasis
}

\author{
Fábio Peixoto $\mathbb{D}^{1,2}$ Maurício T. Nascimento $\left(\mathbb{D},{ }^{1,2}\right.$ Rúbia Costa $\mathbb{D},{ }^{2}$ Juliana Silva $\mathbb{D}^{2}, 2$ \\ Gaby Renard $\mathbb{D}^{3}{ }^{3}$ Luiz Henrique Guimarães $\mathbb{D}^{4},{ }^{4}$ Gerson Penna $\mathbb{D},{ }^{5}$ Manoel Barral-Netto $\mathbb{D}^{1}{ }^{1}$ \\ Lucas P. Carvalho $\mathbb{D}^{1,2,6}$ Paulo R. L. Machado $\mathbb{D}^{2,6}$ and Edgar M. Carvalho $\mathbb{D}^{1,2,6}$ \\ ${ }^{1}$ Instituto Gonçalo Moniz, FIOCRUZ, R. Waldemar Falcão, 121, Candeal, 40296-710 Salvador, BA, Brazil \\ ${ }^{2}$ Serviço de Imunologia, Hospital Universitário Professor Edgard Santos, Universidade Federal da Bahia, R. Dr. Augusto Viana, s/n, \\ Canela, 40301-155 Salvador, BA, Brazil \\ ${ }^{3}$ Quatro G Pesquisa \& Desenvolvimento Ltda, Av. Ipiranga 6681, Prédio 92A, Porto Alegre 90619-900, Brazil \\ ${ }^{4}$ Universidade Federal do Sul da Bahia, Praça Joana Angélica, 58, São Jose, Teixeira de Freitas, 45988 BA, Brazil \\ ${ }^{5}$ Universidade de Brasília (UnB), Núcleo de Medicina Tropical, Brasília, 70910-900 DF, Brazil \\ ${ }^{6}$ Instituto Nacional de Ciência e Tecnologia em Doenças Tropicais (INCT-DT), Salvador, BA, Brazil
}

Correspondence should be addressed to Edgar M. Carvalho; imuno@ufba.br

Received 19 March 2020; Revised 9 July 2020; Accepted 17 July 2020; Published 8 August 2020

Academic Editor: Peirong Jiao

Copyright (C) 2020 Fábio Peixoto et al. This is an open access article distributed under the Creative Commons Attribution License, which permits unrestricted use, distribution, and reproduction in any medium, provided the original work is properly cited.

Cutaneous leishmaniasis (CL) due to L. braziliensis is associated with an exaggerated inflammatory response and tissue damage. Miltefosine is more effective than pentavalent antimony $\left(\mathrm{Sb}^{\mathrm{v}}\right)$ in the treatment of CL, and here, we evaluate the ability of $\mathrm{Sb}^{\mathrm{v}}$, miltefosine, and GM-CSF administered intravenously, orally, or topically, respectively, to modify the immune response. Patients were treated with miltefosine plus GM-CSF, miltefosine plus placebo, or Sb ${ }^{\mathrm{v}}$. Mononuclear cells were stimulated with soluble Leishmania antigen (SLA) on day 0 and day 15 of therapy, and cytokine levels were determined in supernatants by ELISA. The lymphocyte proliferation and oxidative burst were evaluated by flow cytometry, and the degree of infection and Leishmania killing by optical microscopy. Proliferation of $\mathrm{CD}^{+} \mathrm{T}$ cells were enhanced in patients using miltefosine and in $\mathrm{CD} 8^{+} \mathrm{T}$ cells when GM-CSF was associated. Enhancement in the oxidative burst occurred in the miltefosine plus GM-CSF group on day 15 of therapy. Moreover, the number of L. braziliensis in infected monocytes on day 15 as well as the percentage of infected cells was lower after 48- and 72-hour culture in cells from patients treated with miltefosine plus GM-CSF. In addition to the ability of miltefosine to kill Leishmania, the changes in the immune response caused by miltefosine and GM-CSF may increase the cure rate of CL patients using these drugs.

\section{Introduction}

Tegumentary leishmaniasis (TL), caused by the protozoan of the genus Leishmania, is a major health problem in many regions of the world. After being transmitted by sand flies, Leishmania parasites infect human macrophages and dendritic cells (DCs), causing a wide spectrum of clinical manifestations, including visceral leishmaniasis (VL), cutaneous leishmaniasis (CL), disseminated leishmaniasis (DL), mucosal leishmaniasis (ML), and diffuse cutaneous leishmaniasis
(DCL). Cutaneous leishmaniasis (CL) in Latin America is predominantly caused by Leishmania (Viannia) braziliensis and is characterized by the presence of one or a few welldelimited ulcerated lesions with granulomatous fundus and elevated borders [1]. Host immunological factors play an important role in the pathogenesis of the disease. Mononuclear cells from patients with CL stimulated with soluble Leishmania antigens (SLA) display an exacerbated Th1type immune response and produce high levels of IFN- $\gamma$ and TNF and low levels of IL-10 in cultures [2]. The 
production of IFN- $\gamma$ is important to prevent parasite proliferation in mononuclear phagocytes and the dissemination of the infection $[3,4]$. However, this response is not capable of eliminating all the parasites, and the persistent stimulation of the immune system by the parasites and Leishmania antigens leads to an exaggerated inflammatory response resulting in tissue damage [5]. Furthermore, studies have shown a pathogenic role of $\mathrm{CD}^{+} \mathrm{T}$ cells at the lesion site, as the lyses of infected cells release molecules that induce the secretion of IL- $1 \beta$, TNF, inflammasome activation, and the appearance of the ulcer [6]. In this context, inflammatory cytokines may have an important role in parasite eradication, but its overproduction is associated with tissue damage and development of the cutaneous ulcer.

Meglumine antimoniate $\left(\mathrm{Sb}^{\mathrm{v}}\right)$ is the first-choice drug for the treatment of CL in Latin America, but an increase in therapeutic failure, hitting over $50 \%$ of the patients, has been observed in the last 15 years [7, 8]. Other alternative drugs like pentamidine and amphotericin $\mathrm{B}$ have shown toxic activity to patients, and the last one requires hospitalization; therefore, their use is limited. Miltefosine is an oral leishmanicidal drug that can eliminate up to $95 \%$ of $L$. donovani and L. infantum amastigotes in mice [9]. Miltefosine has been effective in the treatment of CL caused by L. braziliensis and L. guyanensis [7, 10]. In Brazil, miltefosine cured 75\% of patients infected with L. braziliensis and $71.4 \%$ of patients infected with L. guyanensis [7, 10].

Although miltefosine is a leishmanicidal drug, its mechanisms of action are not fully understood. It is known that miltefosine acts not only by blocking cytochrome $\mathrm{C}$ oxidase leading to changes in mitochondrial membrane potential and consequently reducing oxygen consumption and ATP levels in L. donovani [11] but also by inhibiting phosphatidylethanolamine N-methyl-transferase and therefore the biosynthesis of phosphatidylcholine [12]. Some studies also demonstrate that miltefosine opens the sphingosineactivated plasma membrane $\mathrm{Ca}^{2+}$ channel and has a direct effect on acidocalcisomes, which in combination result in large intracellular $\mathrm{Ca}^{2+}$ accumulation [13]. Furthermore, miltefosine causes an apoptosis-like death in L. donovani promastigotes [14].

The host immune response has a great impact and influence on the therapeutic response of CL. Patients with diffuse CL, a disease caused by L. amazonensis in Latin America, have a poor Th1-type immune response and are refractory to therapy [15]. Alternatively, the presence of an exaggerated immune response, as observed in CL caused by L. braziliensis, does not prevent the development of the disease [16, 17]. Previous studies showed that $\mathrm{Sb}^{\mathrm{v}}$ associated with granulocyte and macrophage colony stimulation factor (GM-CSF) or pentoxifylline (a drug that decreases TNF production) is more effective and reduces the healing time of CL and ML [18-21]. GM-CSF induces in vitro macrophage activation and increases Leishmania killing [22-24]. Miltefosine has the ability not only to kill Leishmania but also to enhance chemotaxis, motility, monocyte adhesion, and phagocytosis [25]. Studies indicate that the ability of miltefosine to stimulate macrophage and monocyte activation is due to its semblance with phosphatidylcholine, enhancing the membrane fluidness of these cells [25]. The aim of this study was to determine the changes in the immune response in patients treated with intravenous antimony, oral miltefosine, and topical GM-CSF.

\section{Materials and Methods}

2.1. Patients. Participants of this study were patients with CL from the endemic area of Corte de Pedra, Bahia, Brazil, who were participating in an ongoing randomized control study aimed at comparing the efficacy and effectivity of miltefosine associated to GM-CSF versus miltefosine plus placebo and antimoniate of meglumine. The diagnosis of CL was performed by the presence of a typical CL ulcer and detection of DNA of L. braziliensis by PCR. Patients were allocated into three groups. Patients in group 1 received Impavido ${ }^{\mathrm{TM}}$ (Paesel + Lorei, Rheinberg, Germany) at a dose of $2.5 \mathrm{mg} / \mathrm{kg} / \mathrm{d}$ for 28 days with a maximum dose of $150 \mathrm{mg} /$ day orally + topical GM-CSF (gel cream $0.01 \%$, twice a day per 28 days). Patients in group 2 were treated with miltefosine (Impavido) using the same dose and schedule as group $1+$ topical placebo (gel cream twice a day per 28 days). Patients in group 3 received Glucantime (Sanofi-Aventis) at a dose of $20 \mathrm{mg} / \mathrm{kg} / \mathrm{d}$ intravenously for 20 days with a maximum dose of $1200 \mathrm{mg}$. The blood samples of these individuals were collected before treatment (day 0) and during therapy on day 15. Inclusion criteria were age between 18 and 60 years old, illness duration of more than 20 and less than 90 days, and size of the ulcer between 10 and $40 \mathrm{~mm}$.

2.2. Ethical Statement. All patients agreed to participate in the study and signed their informed consent. This study was approved by the Institution Review Board of the Federal University of Bahia Medical School under the number 55647116.2.0000.5577.

\subsection{Immunological Studies}

2.3.1. Separation of Peripheral Blood Mononuclear Cells. Peripheral blood mononuclear cells (PBMC) were obtained from heparinized venous blood by density gradient centrifugation using Ficoll-Hypaque (GE Healthcare). Cells were washed twice in saline and were resuspended at the desired concentration in RPMI 1640 supplemented with 10\% bovine fetal serum (both produced by Gibco, Grand Island, NY, USA) plus antibiotics (complete RPMI).

2.3.2. Cytokine Production Determination. PBMC were adjusted to $3 \times 10^{6} / \mathrm{mL}$ in complete RPMI and cultured in a $37^{\circ} \mathrm{C}, \mathrm{CO}_{2}$ incubator for 72 hours in the presence or absence of SLA $(5 \mu \mathrm{g} / \mathrm{mL})$. The supernatants of those cultures were collected and utilized for the measurement of Granzyme B, IL-1 $\beta$, IL-10, IFN- $\gamma$, TNF, and the chemokines CXCL9 and CXCL10, through ELISA sandwich as previously described.

2.3.3. Lymphocyte Proliferation Essay. To evaluate lymphocyte proliferation, $1 \times 10^{6}$ PBMC were cultured in the presence or absence of SLA $(5 \mu \mathrm{g} / \mathrm{mL})$. After 5 days of incubation at $37^{\circ} \mathrm{C}$ with $5 \% \mathrm{CO}_{2}$, cells were marked with the conjugated antibodies anti-CD4 and anti-CD8, with the 
goal of separating the lymphocyte subpopulations, and antiKi-67 as a cell proliferation marker. Afterwards, cells were analyzed by flow cytometry and data were analyzed by FlowJo ${ }^{\circledR}$. Cells stimulated with $\alpha \mathrm{CD} 3+\alpha \mathrm{CD} 28$ were the positive control.

2.3.4. Oxidative Burst Quantification. To evaluate the reactive oxygen species, $1 \times 10^{6} \mathrm{PBMC}$ were treated with dihydrorhodamine-123 at $10 \mathrm{ng} / \mathrm{mL}$ (Cayman Chemical Company) for 10 minutes. After that, cells were infected with Leishmania braziliensis (Lb), 5 Leishmania parasites per monocyte, considering that this cell type is $15 \%$ of the total PBMC, for 25 minutes, and then stained with anti-HLADR and anti-CD14 fluorochrome-conjugated antibodies. The fluorescence intensity of the cells was assessed by flow cytometry, and data were analyzed through FlowJo ${ }^{\circledR}$. Positive controls were cells stimulated with phorbol myristate acetate.

2.3.5. Infection with L. braziliensis. To evaluate the infection rate, $2.5 \times 10^{6}$ cells $/ \mathrm{mL}$ were incubated in $\mathrm{Nunc}^{\circledR}$ Lab-Tek $^{\circledR}$ plates for 2 hours for monocyte adhesion, and the nonadherent cells were washed out of the plate. Adherent cells (90\% monocytes) were infected with $L$. braziliensis (5 parasites per monocyte) for two hours, and the remaining promastigotes were washed out of the plate. Cells were cultured for 2,48 , or 72 hours at $37^{\circ} \mathrm{C}$ with $5 \% \mathrm{CO}_{2}$. The slides were stained with panoptic stain for quantification of infected monocytes and the number of amastigotes per 100 monocytes was determined by optical microscopy.

2.4. Statistical Analysis. Mann-Whitney's test was used for comparisons between 2 independent continuous variables, Wilcoxon's $U$ test was used for continuous dependent variables, and the Kruskal-Wallis's test and Dunn's posttest were used to compare 3 continuous variables. $p<0.05$ was considered statistically significant, and all $p$ values represented are 2-tailed. All experiments were statically analyzed through Prism GraphPad ${ }^{\circledR}$ 8.0.2, such as the graphics elaboration.

\section{Results}

3.1. Cytokine Production. PBMC from CL patients treated with miltefosine plus GM-CSF, miltefosine plus placebo, or $\mathrm{Sb}^{\mathrm{v}}$, were stimulated with SLA before and on day 15 of therapy, and the production of Granzyme B, IFN- $\gamma$, TNF, IL- $1 \beta$, IL-10, CXCL9, and CXCL10 were compared among the groups. Granzyme B production (Figure 1(a)) in patients treated with miltefosine plus GM-CSF decreased from $2888 \mathrm{pg} / \mathrm{mL}(1654-3111 \mathrm{pg} / \mathrm{mL})$ in day 0 to 1401 (436$2564 \mathrm{pg} / \mathrm{mL})$ in day $15,(p=0.0001)$. The same effect was observed in miltefosine plus placebo-treated patients in which Granzyme B production decreased from $2574 \mathrm{pg} / \mathrm{mL}$ $(418-3219 \mathrm{pg} / \mathrm{mL})$ in day 0 to $1657(650.2-1998 \mathrm{pg} / \mathrm{mL})$ on day $15(p=0.0021)$. When comparing groups among each other during treatment (day 15), we could observe lower levels of this protein in the miltefosine plus GM-CSF group than in the miltefosine plus placebo $(p=0.01)$ and meglumine antimoniate groups, $2183 \mathrm{pg} / \mathrm{mL}(1810-3102 \mathrm{pg} / \mathrm{mL})$ $(p<0.0001)$. Regarding IFN- $\gamma$ (Figure 1(b)), patients treated with miltefosine plus GM-CSF presented a higher level on day 15 of therapy, $1627 \mathrm{pg} / \mathrm{mL}(162-6351 \mathrm{pg} / \mathrm{mL})$, than patients who received miltefosine plus placebo, $433.5 \mathrm{pg} / \mathrm{mL}$ $(0-9739 \mathrm{pg} / \mathrm{mL}) \quad(p=0.048), \quad$ or $\quad \mathrm{Sb}^{\mathrm{v}}, \quad 239 \mathrm{pg} / \mathrm{mL} \quad(0-$ $742 \mathrm{pg} / \mathrm{mL}) \quad(p=0.0032)$. The TNF level (Figure 1(c)) observed in patients inserted in the same treatment group (MF plus GM-CSF) was $654 \mathrm{pg} / \mathrm{mL}(244-3127 \mathrm{pg} / \mathrm{mL}$ ), and the TNF level in patients treated with miltefosine plus placebo was $879 \mathrm{pg} / \mathrm{mL}(54-2500 \mathrm{pg} / \mathrm{mL})$, which were higher than those found in patients treated with $\mathrm{Sb}^{\mathrm{v}}, 382 \mathrm{pg} / \mathrm{mL}$ $(0-1550 \mathrm{pg} / \mathrm{mL})(p=0.03$ and $p=0.046)$. The IL- $\beta$ production (Figure $1(\mathrm{~d})$ ) in patients treated with miltefosine plus GM-CSF increased from $38 \mathrm{pg} / \mathrm{mL}(8-92 \mathrm{pg} / \mathrm{mL})$ on day 0 to $128 \mathrm{pg} / \mathrm{mL}(26-300 \mathrm{pg} / \mathrm{mL})(p=0.01)$. Neither of the other treatment options modified this cytokine production. The level of CXCL10 (Figure 1(e)) was higher in the miltefosine plus GM-CSF group, $1687 \mathrm{pg} / \mathrm{mL}(1177-20000 \mathrm{pg} / \mathrm{mL})$, than in the $\mathrm{Sb}^{\mathrm{v}}$ group, $1384.5 \mathrm{pg} / \mathrm{mL}(15-2087 \mathrm{pg} / \mathrm{mL})(p=0.03)$. Regarding IL-10 and CXCL9, no statistical difference has been observed.

3.2. Lymphocyte Proliferation. PBMC were cultured for 5 days for the evaluation of $\mathrm{CD} 4^{+}$and $\mathrm{CD} 8^{+} \mathrm{T}$ cell proliferation through Ki-67 expression. To accomplish that, we quantified the frequency of $\mathrm{T}$ cells expressing this molecule in day 15 and divided this by the frequency found in day 0 , allowing the analysis of drug capacity in inducing proliferation. Using this index, we observed an increase in $\mathrm{CD} 4^{+} \mathrm{T}$ proliferation in patients treated with miltefosine plus GM-CSF and miltefosine plus placebo, whereas a decrease in the $\mathrm{Sb}^{\mathrm{v}}$ group was noted. The same procedure was done with $\mathrm{CD}^{+} \mathrm{T}$ cells and, with miltefosine plus GM-CSF as an exception, a decrease in proliferation was observed during the treatment (Figure 2(b)).

3.3. Oxidative Burst. The reactive oxygen species (ROS) produced by monocytes from CL patients after 25 minutes of infection with $L$. braziliensis before and on day 15 of therapy is shown in Figure 3. The median fluorescence index (MFI) of DHR 123 of monocytes from patients treated with miltefosine plus GM-CSF enhanced from 25 (8-31) to 44.5 (19-81) from day 0 to day $15(p=0.03)$ (Figure $3(\mathrm{~b})$ ). There was no difference in the oxidative burst during therapy in the other groups.

3.4. Infection Rate and Parasite Killing. Monocytes were isolated through adhesion in Nunc ${ }^{\circledR}$ Lab-Tek $^{\circledR}$ chambers, infected with $L$. braziliensis, and cultured for 2, 48, and 72 hours. After 2 hours of incubation, we observed that monocytes from miltefosine plus GM-CSF and miltefosine plus placebo-treated patients had higher infection rate on day 15 of therapy than before treatment, 49 (45-53) versus 40 (3943) $(p=0.0079)$ and $47(43-50)$ versus $42(40-45)(p=0.02)$ (Figure 4(a)), as well as a higher number of amastigotes internalized per 100 monocytes, 266 (205-277) versus 181 (169201) $(p=0.008)$ and $203(196-222)$ versus $169(155-197)$ $(p=0.015)$ (Figure $4(\mathrm{~d}))$. When monocytes were obtained on day 15 and were cultured for 48 hours, we observed a decrease in the infection ratio in the miltefosine plus GMCSF group, $49(44-53)$ versus $56(55-60)$ at day $0(p=0.03)$ 


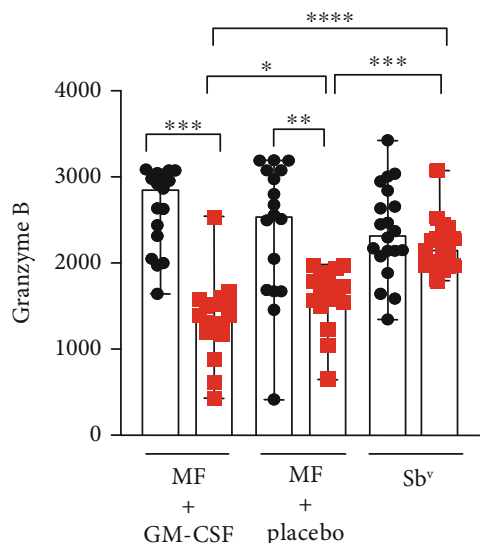

- Day 0

- Day 15

(a)

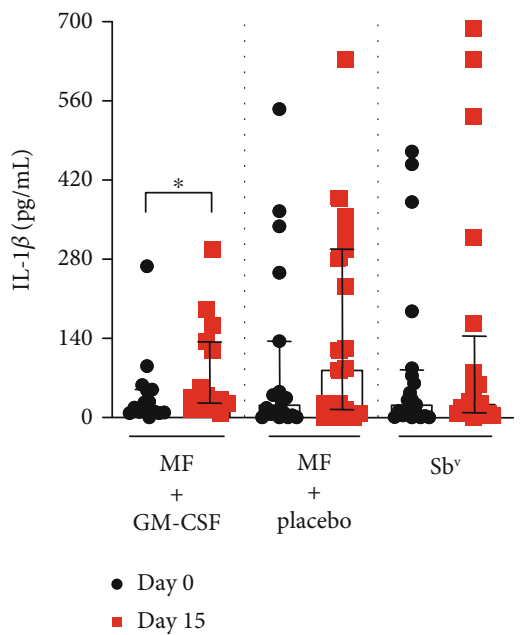

(d)

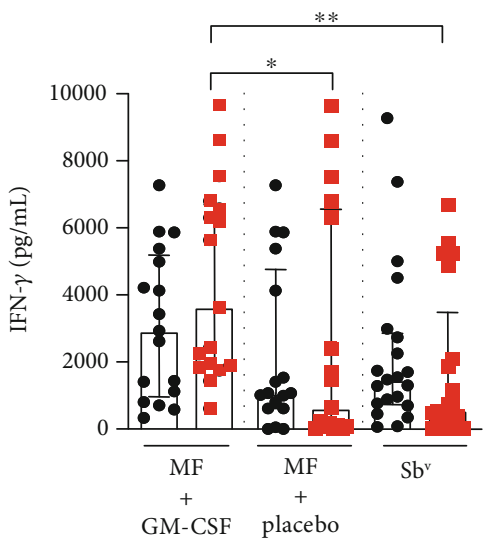

- Day 0

- Day 15

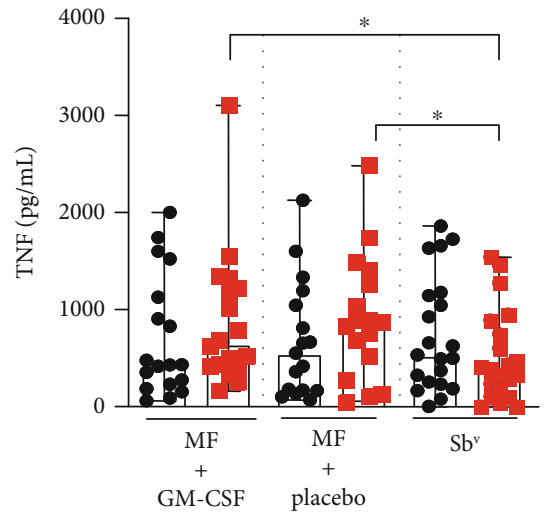

- Day 0

- Day 15

(c)
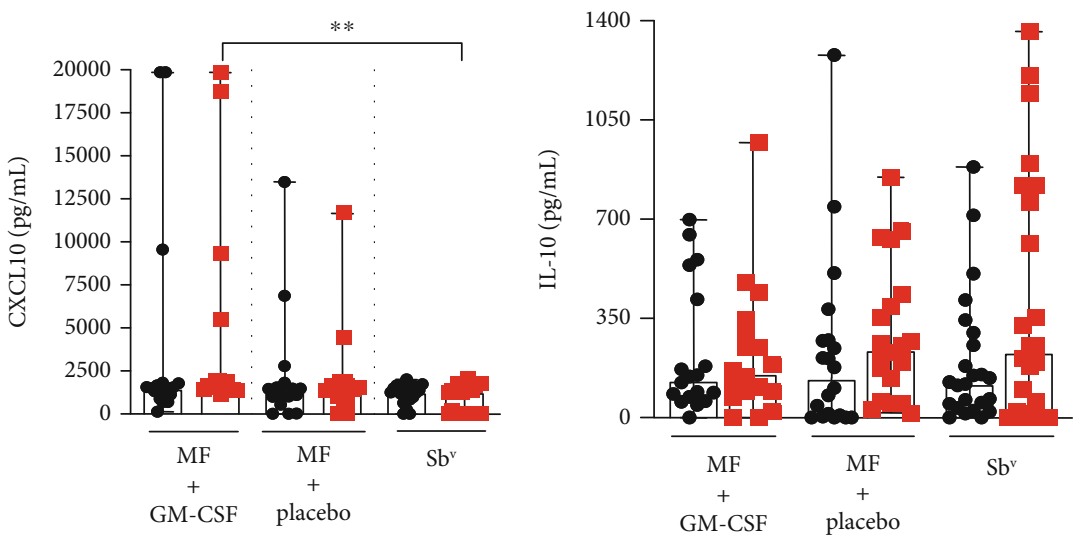

- Day 0

- Day 15

- Day 0

- Day 15

(f)

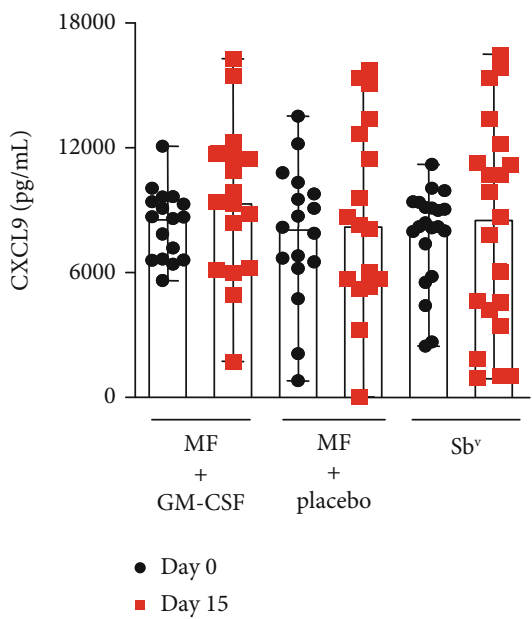

(g)

FIGURE 1: Cytokine production by PBMC from CL patients during therapy. PBMC from patients treated with miltefosine + GM-CSF ( $n=17)$, miltefosine + placebo $(n=17)$ and $\mathrm{Sb}^{\mathrm{v}}(n=21)$ were stimulated with SLA $(5 \mu \mathrm{g} / \mathrm{mL})$ for 72 hours on day 0 and 15 of therapy. (a) Granzyme B, (b) IFN- $\gamma$, (c) TNF, (d) IL-1 $\beta$, (e) CXCL10, (f) IL-10, and (g) CXCL9 levels were determined in culture supernatants by ELISA. The data is represented with median and interquartile range. Statistical analyses were performed using Wilcoxon's or Mann-Whitney's rank test; ${ }^{*} p<0.05,{ }^{* *} p<0.01,{ }^{* * *} p<0.001$, and ${ }^{* * * *} p<0.0001$. 

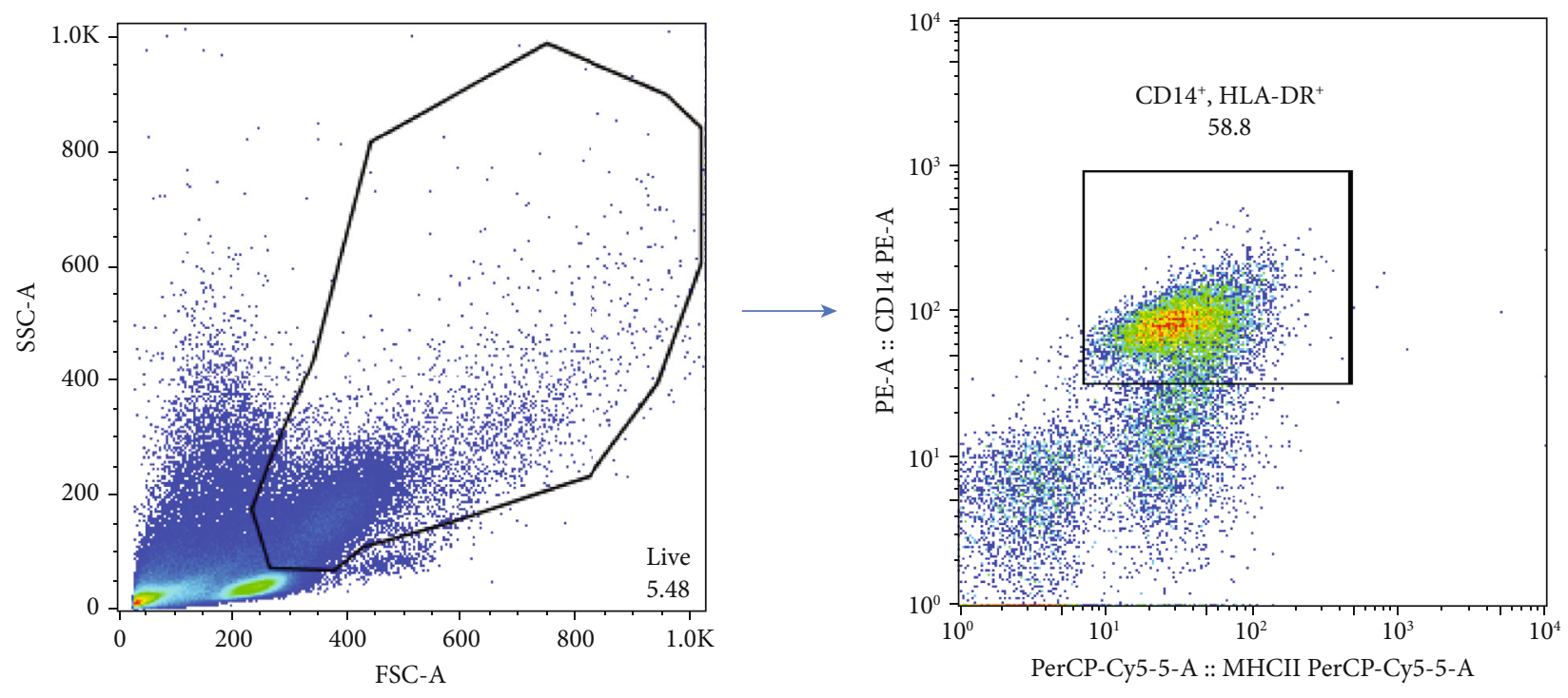

(a)

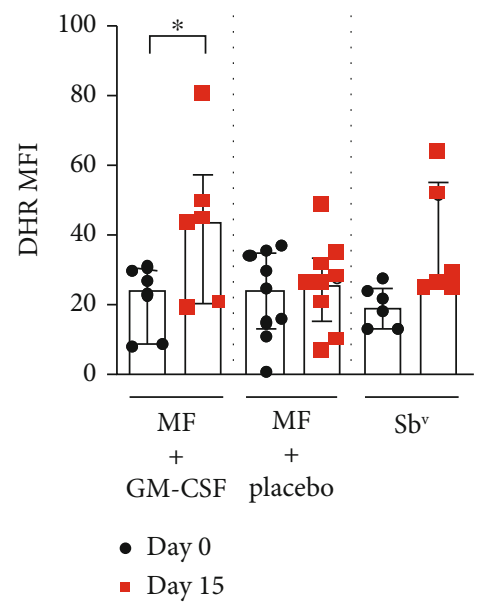

(b)

Figure 2: Miltefosine plus GM-CSF treatment enhances reactive oxygen species production by monocytes after L. braziliensis infection. Monocytes from CL patients were evaluated on day 0 and 15 of treatment with miltefosine + GM-CSF $(n=6)$, miltefosine + placebo $(n=9)$, and $\mathrm{Sb}^{\mathrm{v}}(n=6)$. The cells were treated with DHR $(10 \mathrm{ng} / \mathrm{mL}-10 \mathrm{~min})$ and infected with L. braziliensis promastigotes for 25 minutes at a 5:1 ratio. Cells were stained with anti-CD14 and anti-HLA-DR. Data were collected using flow cytometry and analyzed using FlowJo ${ }^{\circledR}$ software. (a) Representative gating strategy on $\mathrm{CD}_{14}{ }^{+}$and HLA-DR ${ }^{+}$expression in monocytes from one CL patient. DHR MFI was taken from CD14 ${ }^{+} \mathrm{HLA}_{-} \mathrm{DR}^{+}$population. (b) The data represent the mean of fluorescence intensity (MFI) of oxidative burst production by monocytes from CL patients inserted in the treatment groups. The data is represented with median and interquartile ranges. Statistical analyses were performed using Mann-Whitney's test for unpaired groups and Wilcoxon's rank test for paired measurements; ${ }^{*} p<0.05$.

(Figure 4(b)), and a decrease after 72 hours by both the miltefosine plus GM-CSF-treated group, 39 (36/40) in day 15 versus $45(43 / 49)$ in day $0(p=0.008)$, and the miltefosine plus placebo-treated group $(p=0.009)$ (Figure $4(c)$ ). The same phenomena were observed when comparing the number of amastigotes during versus before therapy. At day 15 of therapy, when monocytes were cultured for 48 hours, there was a decrease in parasites internalized by monocytes in the miltefosine plus GM-CSF group $(p=0.03)$ (Figure 4(e)); when monocytes were cultured for 72 hours, there was a similar decrease in internalized parasites in the miltefosine plus GM-CSF-treated group from 153 (139-162) to 114 (90-133) $(p=0.008)$. The same was observed in patients treated with miltefosine plus placebo $(p=0.008)$ (Figure $4(\mathrm{f})$ ). When comparing groups, we observed a higher percentage of infected cells in the miltefosine plus GM-CSF group compared to antimony, $49(45-53)$ versus $40(37-47)(p=0.02)$ (Figure 4(a)). We also observed a higher number of amastigotes internalized by monocytes from the first group of patients, 266 (205-277), compared to the miltefosine plus placebo group $(p=0.03)$ and the $\mathrm{Sb}^{\mathrm{v}}$ group, $176(166-193)$ $(p=0.007)$, after 2 hours of incubation (Figure $4(d))$. The miltefosine plus placebo group also presented higher levels of amastigote internalization than meglumine antimoniate- 


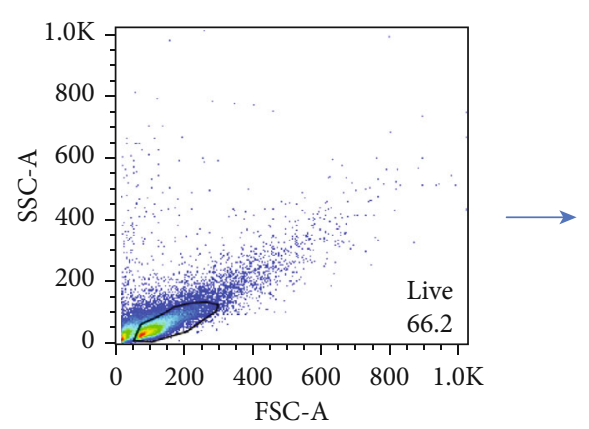

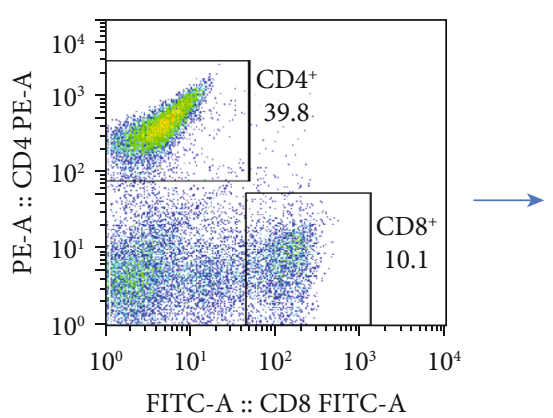

(a)

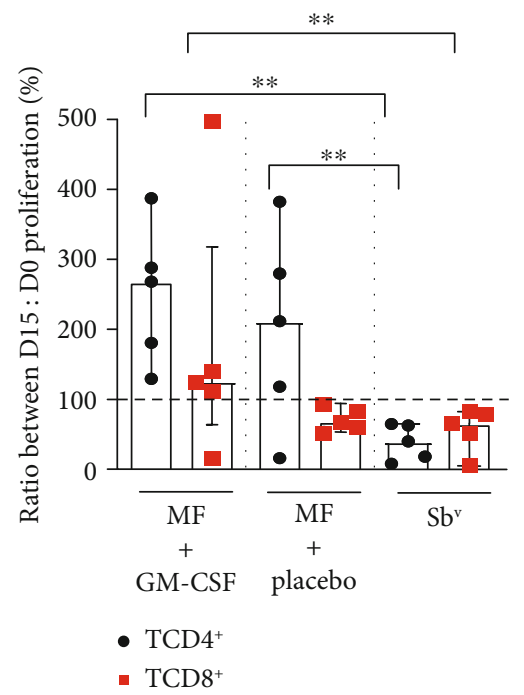

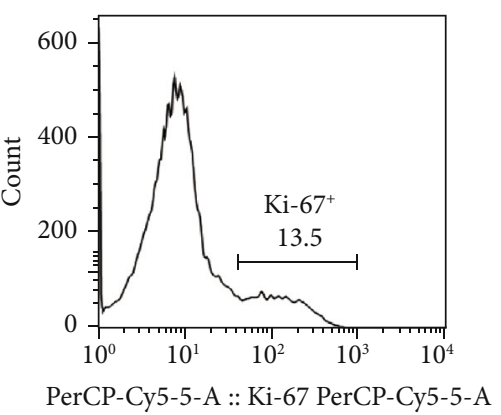

PerCP-Cy5-5-A :: Ki-67 PerCP-Cy5-5-A

(b)

Figure 3: Miltefosine treatment induces $\mathrm{CD} 4^{+} \mathrm{T}$ cell proliferation, and its association with GM-CSF enhances CD ${ }^{+} \mathrm{T}$ cell proliferation by

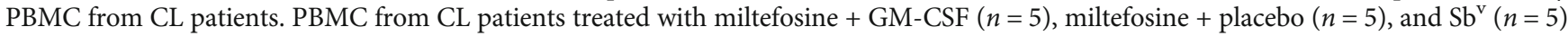
were cultured for 5 days in the presence of SLA on day 0 and 15 of therapy. Cells were stained with anti-CD4, anti-CD8, and anti-Ki67. Data were collected using flow cytometry and analyzed by FlowJo ${ }^{\circledR}$ software. (a) Representative gating strategy on $\mathrm{CD} 4^{+}$, $\mathrm{CD} 8^{+}$, and $\mathrm{Ki}-67^{+}$ expression in lymphocytes from one CL patient. (b) The data represent the ratio between the proliferation found at day 15 and day 0 of treatment from CL patients inserted in the treatment groups added by 100 . The data is represented with median and interquartile range. Statistical analyses were performed using Mann-Whitney's test for unpaired groups and Wilcoxon's rank test for paired measurements; ${ }^{*} p<0.05$ and ${ }^{* *} p<0.01$.

treated patients $(p=0.008)$ (Figure $4(\mathrm{~d})$ ). After 48 hours, the relation between the miltefosine plus GM-CSF group and the antimony group is inverted, and patients from the second group presented a higher percentage of infected cells $(p=0.016)$ (Figure $4(\mathrm{~b}))$ as well as a higher number of amastigotes per 100 monocytes $(p=0.015)$ (Figure 4(e)). Analyzing 72-hour cultures, we saw a lower infection ratio in both the miltefosine plus GM-CSF group, 39 (36-40), and the miltefosine plus placebo group, 39 (35-42), compared to antimony-treated patients, $43(42-46)(p=0.007$ and $p=$ 0.02 ) (Figure $4(\mathrm{c})$ ), as well as lower number of amastigotes internalized, 114 (90-133) and 124 (105-146) versus 160 $(140-171)(p=0.007$ and $p=0.016)$, respectively (Figure $4(\mathrm{f})$ ).

\section{Discussion}

Miltefosine is effective against visceral and cutaneous leishmaniasis, and the cure rate of this drug is higher than that observed with meglumine antimoniate in ATL (American Tegumentary Leishmaniasis). Miltefosine also has immunomodulatory properties as it increases phagocytosis and enhances IFN- $\gamma$ production, the main cytokine that activates macrophages for Leishmania killing [7, 26-28]. GM-CSF has a wide effectivity on monocytes and macrophages, activating these cells and granting a leishmanicidal effect [22-24]. Furthermore, in mice infected with Mycobacterium tuberculosis, this molecule inducts the recruitment of macrophages and lymphocytes to the lesion site [29]. We have previously shown that GM-CSF associated to $\mathrm{Sb}^{\mathrm{v}}$ increases the cure rate and reduces the healing time of cutaneous leishmaniasis [19]. In the present study, taking advantage of an ongoing clinical trial evaluating the efficacy of miltefosine plus GM-CSF vs. miltefosine plus placebo vs. $\mathrm{Sb}^{\mathrm{v}}$, we compared the immunological response of CL patients before and during therapy. We observed that, in vitro, monocytes from patients using miltefosine plus GM-CSF increased the respiratory burst 


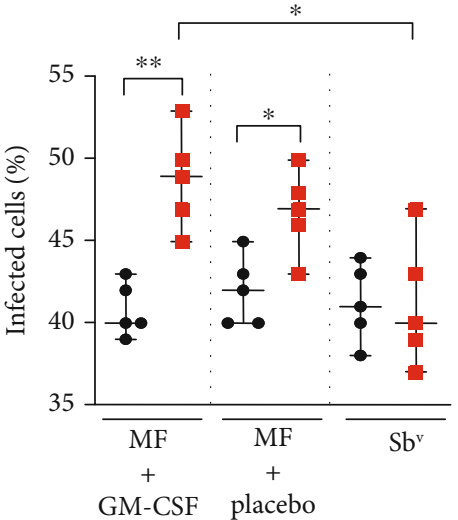

- Day 0

- Day 15

(a)

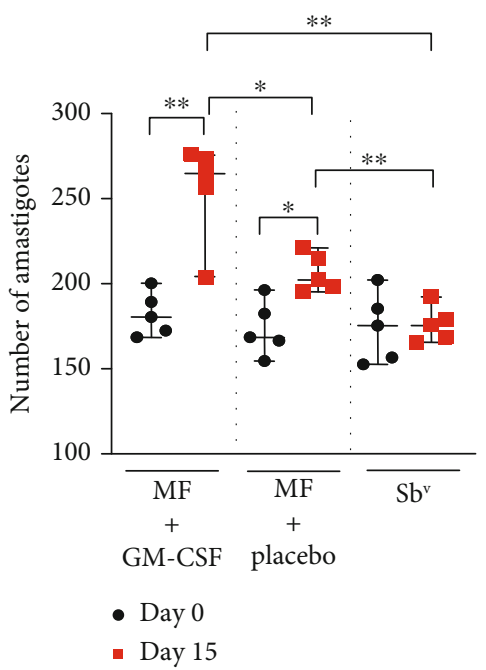

(d)

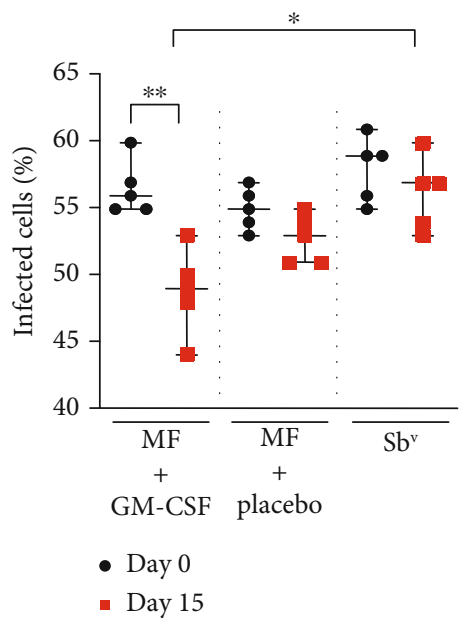

(b)

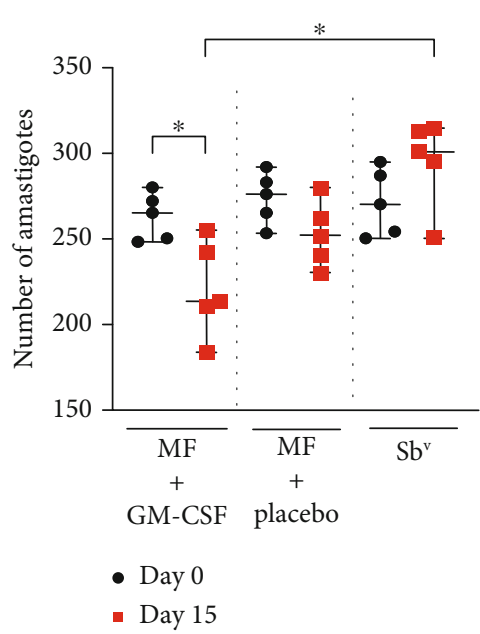

(e)

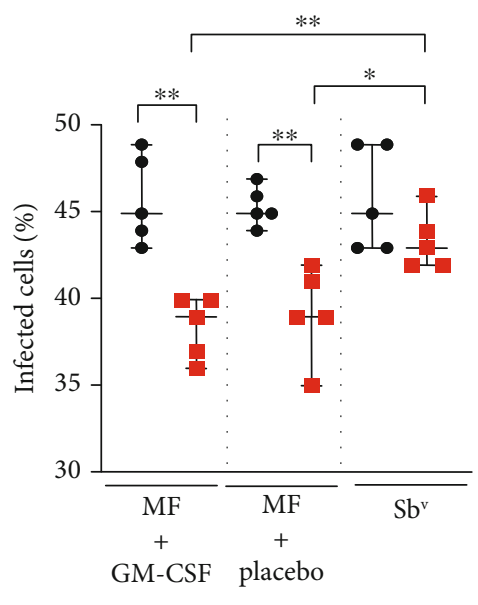

- Day 0

- Day 15

(c)

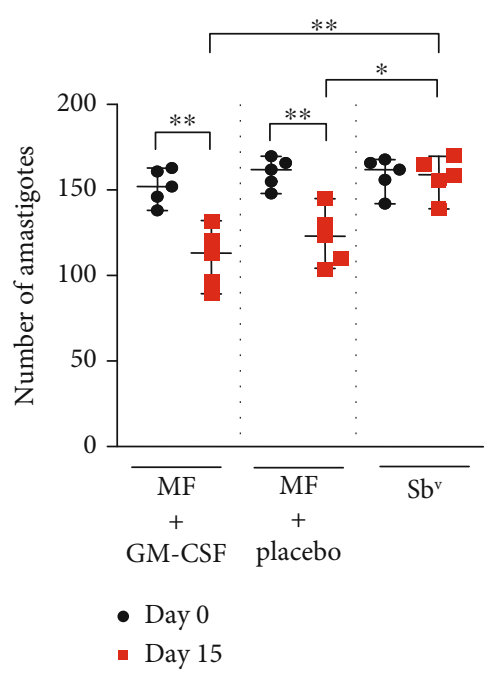

(f)

FIGURE 4: Influence of miltefosine and GM-CSF treatment in the phagocytosis and killing of L. braziliensis by monocytes from CL patients. Monocytes from CL patients treated with miltefosine + GM-CSF $(n=5)$, miltefosine + placebo $(n=5)$, and Sb ${ }^{\mathrm{v}}(n=5)$ were infected with $L$. braziliensis promastigotes at a $5: 1$ ratio for 2,48 , and 72 hours. The percentage of infected cells after 2 hours (a), 48 hours (b), and 72 hours (c) as well as the number of intracellular parasites after 2 (d), 48 (e), and 72 (f) hours were determined by optical microscopy, after panoptic staining on day 0 and day 15 of therapy. The data is represented with median and interquartile range. Statistical analyses were performed using Mann-Whitney's test for unpaired groups and Wilcoxon's rank test for paired measurements; ${ }^{*} p<0.05,{ }^{* *} p<0.01$.

and decreased the percentage of infected cells as well as the number of amastigotes per 100 monocytes during treatment. Moreover, there was an increase in the percentage of $\mathrm{CD} 4^{+}$ and $\mathrm{CD}^{+} \mathrm{T}$ cell proliferation, an increase in IL- $1 \beta$ production, and a decrease of Granzyme B concentration.

The control of Leishmania infection is associated with a Th1 immune response. $\mathrm{CD}^{+} \mathrm{T}$ cell activation and, consequently, IFN- $\gamma$ production participate in host protection, and in this study, we showed that miltefosine increases the number of $\mathrm{CD}^{+} \mathrm{T}$ cells, which may favor parasite killing and release of Leishmania antigens and, consequently, more lymphocyte activation. In addition to an increase in $\mathrm{CD} 4^{+}$ $\mathrm{T}$ cells, miltefosine therapy kept the enhancement of IFN- $\gamma$ and TNF that are cytokines already enhanced during L. braziliensis infection.

Previous studies showed that IFN- $\gamma$ is necessary for the control of parasite proliferation, granting leishmanicidal effects by mononuclear phagocytes $[3,4]$. Thus, it is possible that, in addition of the leishmanicidal effect of miltefosine, the maintenance of IFN- $\gamma$ levels may also contribute to parasite killing and a higher cure rate in patients treated with miltefosine plus GM-CSF and miltefosine plus placebo than in those treated with meglumine antimoniate $[6,8]$.

In addition to IFN- $\gamma$ and TNF, other cytokines are highly produced during CL and some of them have been associated with the pathogenesis of the disease. CXCL9 and CXCL10 are 
produced in high levels during L. braziliensis infection and are associated with the chemotaxis of T and NK cells to the lesion site [30]. Alternatively, IL-10, a regulatory cytokine, may favor parasite proliferation but may attenuate tissue damage during Leishmania infection [31]. However, we did not observe any important influence of the drugs used in modifying the production of these molecules.

Recently, emphasis has been given to the role of cytotoxic $\mathrm{CD}^{+} \mathrm{T}$ cells and IL- $1 \beta$ production in the pathology of CL. $\mathrm{CD}^{+} \mathrm{T}$ cells from CL patients display a higher expression of Granzyme B, and while they kill Leishmania-infected cells, they have a limited ability to kill L. braziliensis [32-34]. Moreover, it has been suggested that cell killing activates inflammasome and IL- $1 \beta$ production and this inflammatory response leads to tissue damage and ulcer development $[33,35]$. Here, we showed that miltefosine decreased Granzyme B production which may have contributed to a decreased pathology. Patients who received miltefosine, as well as $\mathrm{Sb}^{\mathrm{v}}$, did not modify IL- $1 \beta$ production, but those treated with miltefosine plus GM-CSF had enhanced IL- $1 \beta$ levels. However, while cytotoxicity mediated by $\mathrm{CD}^{+} \mathrm{T}$ cells and Granzyme B have been associated with pathology in CL, IL- $1 \beta$ seems to have a dual effect as this cytokine may have a protective role in the early phase of the infection in mice [36].

Previous studies have shown that miltefosine changes the membrane fluidity of monocytes and macrophages, which might enhance phagocytic function by these cells [25]. When those cells were stimulated in vitro with miltefosine and cultured with Saccharomyces cerevisiae, the drug enhanced phagocytosis by macrophages as well as the number of cells engaged in this activity [27]. Here, we show for the first time that miltefosine enhances the phagocytosis of L. braziliensis, and more importantly, we documented a decrease in the frequency of infected cells as well as in the number of amastigotes after 48 and 72 hours of culture of monocytes from patients treated with miltefosine when compared to monocytes before treatment and also with cells from patients treated with meglumine antimoniate. Leishmania killing by macrophages is dependent on the respiratory burst with an increase in the production of reactive oxygen species [37]. During CL by L. braziliensis infection, the killing is predominantly performed by classic macrophages [35]. While in the mouse model of CL, NO production has been associated with Leishmania killing, in humans, we found no leishmanicidal role for this molecule. Instead, inhibition of ROS production decreased the ability of human monocytes to kill Leishmania [37]. Our observation that miltefosine enhances the oxidative burst indicates that in addition of inducing phagocytosis, the drug increases parasite killing.

\section{Conclusions}

Our results confirm that miltefosine enhances monocytic function and also show that this drug enhances IL- $1 \beta$ production and maintains the levels of IFN- $\gamma$ and TNF observed before therapy. We also showed that topical use of GM-CSF associated with oral miltefosine modifies the systemic immune response increasing IFN- $\gamma$ and ROS production and decreasing Granzyme B levels. This observation may contribute to better parasite control and an increase in the cure rate of patients with cutaneous leishmaniasis treated with miltefosine.

\section{Data Availability}

The data used to support the findings of this study have been deposited in the figshare repository (DOI 10.6084/m9 .figshare.12003192) and are included within the article.

\section{Conflicts of Interest}

The authors declare that there is no conflict of interest regarding the publication of this paper.

\section{Acknowledgments}

We would like to thank Ednaldo Lago and Fernanda Ventim for field assistance and Thiago Cardoso for guidance and help with the figures. This study was sponsored by the Brazilian Ministry of Health and the National Council for Scientific and Technological Development (CNPq).

\section{References}

[1] A. L. Bittencourt and A. Barral, "Evaluation of the histopathological classifications of American cutaneous and mucocutaneous leishmaniasis," Memorias do Instituto Oswaldo Cruz, vol. 86, no. 1, pp. 51-56, 1991.

[2] O. Bacellar, H. Lessa, A. Schriefer et al., "Up-regulation of Th1type responses in mucosal leishmaniasis patients," Infection and Immunity, vol. 70, no. 12, pp. 6734-6740, 2002.

[3] A. Ribeiro-de-Jesus, R. P. Almeida, H. Lessa, O. Bacellar, and E. M. Carvalho, "Cytokine profile and pathology in human leishmaniasis," Brazilian Journal of Medical and Biological Research., vol. 31, no. 1, pp. 143-148, 1998.

[4] F. Y. Liew, Y. Li, and S. Millott, "Tumor necrosis factor-alpha synergizes with IFN-gamma in mediating killing of Leishmania major through the induction of nitric oxide," The Journal of Immunology, vol. 145, no. 12, pp. 4306-4310, 1990.

[5] L. P. Carvalho, S. Passos, A. Schriefer, and E. M. Carvalho, "Protective and pathologic immune responses in human tegumentary leishmaniasis," Frontiers in Immunology, vol. 3, p. 301, 2012.

[6] F. O. Novais and P. Scott, "CD8 ${ }^{+} \mathrm{T}$ cells in cutaneous leishmaniasis: the good, the bad, and the ugly," Seminars in Immunopathology, vol. 37, no. 3, pp. 251-259, 2015.

[7] P. R. Machado, J. Ampuero, L. H. Guimarães et al., "Miltefosine in the treatment of cutaneous leishmaniasis caused by Leishmania braziliensis in Brazil: a randomized and controlled trial," PLOS Neglected Tropical Diseases, vol. 4, no. 12, article e912, 2010.

[8] F. V. Prates, M. E. Dourado, S. C. Silva et al., "Fluconazole in the treatment of cutaneous leishmaniasis caused by Leishmania braziliensis: a randomized controlled trial," Clinical Infectious Diseases, vol. 64, no. 1, pp. 67-71, 2016.

[9] A. Kuhlencord, T. Maniera, H. Eibl, and C. Unger, "Hexadecylphosphocholine: oral treatment of visceral leishmaniasis in mice," Antimicrobial Agents and Chemotherapy, vol. 36, no. 8, pp. 1630-1634, 1992. 
[10] A. Chrusciak-Talhari, R. Dietze, C. C. Talhari et al., "Randomized controlled clinical trial to access efficacy and safety of miltefosine in the treatment of cutaneous leishmaniasis caused by Leishmania (Viannia) guyanensis in Manaus, Brazil," The American Journal of Tropical Medicine and Hygiene, vol. 84, no. 2, pp. 255-260, 2011.

[11] J. R. Luque-Ortega and L. Rivas, "Miltefosine (hexadecylphosphocholine) inhibits cytochrome $c$ oxidase in Leishmania donovani promastigotes," Antimicrobial Agents and Chemotherapy, vol. 51, no. 4, pp. 1327-1332, 2007.

[12] M. Rakotomanga, S. Blanc, K. Gaudin, P. Chaminade, and P. M. Loiseau, "Miltefosine affects lipid metabolism in Leishmania donovani promastigotes," Antimicrobial Agents and Chemotherapy, vol. 51, no. 4, pp. 1425-1430, 2007.

[13] A. K. Pinto-Martinez, J. Rodriguez-Durán, X. Serrano-Martin, V. Hernandez-Rodriguez, and G. Benaim, "Mechanism of action of miltefosine on Leishmania donovani involves the impairment of acidocalcisome function and the activation of the sphingosine-dependent plasma membrane $\mathrm{Ca}^{2+}$ channel," Antimicrobial Agents and Chemotherapy, vol. 62, no. 1, 2018.

[14] C. Paris, P. M. Loiseau, C. Bories, and J. Bréard, "Miltefosine induces apoptosis-like death in Leishmania donovani promastigotes," Antimicrobial Agents and Chemotherapy, vol. 48, no. 3, pp. 852-859, 2004.

[15] J. Convit, M. Ulrich, C. T. Fernandez et al., "The clinical and immunological spectrum of American cutaneous leishmaniasis," Transactions of the Royal Society of Tropical Medicine and Hygiene., vol. 87, no. 4, pp. 444-448, 1993.

[16] R. S. Costa, L. P. Carvalho, T. M. Campos et al., "Early cutaneous leishmaniasis patients infected with Leishmania braziliensis express increased inflammatory responses after antimony therapy," Journal of Infectious Diseases, vol. 217, no. 5, pp. 840-850, 2018.

[17] A. Unger, S. O’Neal, P. R. Machado et al., “Association of treatment of American cutaneous leishmaniasis prior to ulcer development with high rate of failure in northeastern Brazil," The American Journal of Tropical Medicine and Hygiene, vol. 80, no. 4, pp. 574-579, 2009.

[18] H. A. Lessa, P. Machado, F. Lima et al., "Successful treatment of refractory mucosal leishmaniasis with pentoxifylline plus antimony," The American Journal of Tropical Medicine and Hygiene, vol. 65, no. 2, pp. 87-89, 2001.

[19] R. P. Almeida, J. Brito, P. L. Machado et al., "Successful treatment of refractory cutaneous leishmaniasis with GM-CSF and antimonials," The American Journal of Tropical Medicine and Hygiene, vol. 73, no. 1, pp. 79-81, 2005.

[20] G. Brito, M. Dourado, L. Polari et al., "Clinical and immunological outcome in cutaneous leishmaniasis patients treated with pentoxifylline," The American Journal of Tropical Medicine and Hygiene, vol. 90, no. 4, pp. 617-620, 2014.

[21] P. R. L. Machado, H. Lessa, M. Lessa et al., "Oral pentoxifylline combined with pentavalent antimony: a randomized trial for mucosal leishmaniasis," Clinical Infectious Diseases, vol. 44, no. 6, pp. 788-793, 2007.

[22] W. Y. Weiser, A. N. Van Niel, S. C. Clark, J. R. David, and H. G. Remold, "Recombinant human granulocyte/macrophage colonystimulating factor activates intracellular killing of Leishmania donovani by human monocyte-derived macrophages," Journal of Experimental Medicine, vol. 166, no. 5, pp. 1436-1446, 1987.

[23] J. L. Do, S. G. Reed, E. A. Wick, and M. Giordano, “Granulocyte-macrophage and macrophage colony-stimulating factors activate intramacrophage killing of Leishmania mexicana amazonensis," Journal of Infectious Diseases, vol. 162, no. 1, pp. 224-230, 1990.

[24] F. Al-Zamel, F. J. Al-Shammary, S. El-Shewemi, and R. Soliman, "Enhancement of leishmanicidal activity of human macrophages against Leishmania major and Leishmania donovani infection using recombinant human granulo," Zentralblatt für Bakteriologie, vol. 285, no. 1, pp. 92-105, 1996.

[25] R. Zeisig, M. Rudolf, I. Eue, and D. Arndt, "Influence of hexadecylphosphocholine on the release of tumor necrosis factor and nitroxide from peritoneal macrophages in vitro," Journal of Cancer Research and Clinical Oncology, vol. 121, no. 2, pp. 69-75, 1995.

[26] T. K. Jha, S. Sundar, C. P. Thakur et al., "Miltefosine, an oral agent, for the treatment of Indian visceral leishmaniasis," New England Journal of Medicine, vol. 341, no. 24, pp. 17951800, 1999.

[27] C. B. Ponte, É. A. R. Alves, R. N. R. Sampaio et al., "Miltefosine enhances phagocytosis but decreases nitric oxide production by peritoneal macrophages of C57BL/6 mice," International Immunopharmacology, vol. 13, no. 1, pp. 114-119, 2012.

[28] P. Wadhone, M. Maiti, R. Agarwal, V. Kamat, S. Martin, and B. Saha, "Miltefosine promotes IFN- $\gamma$-dominated antileishmanial immune response," The Journal of Immunology, vol. 182, no. 11, pp. 7146-7154, 2009.

[29] S. Benmerzoug, F. V. Marinho, S. Rose et al., "GM-CSF targeted immunomodulation affects host response to M. tuberculosis infection," Scientific Reports, vol. 8, no. 1, article 8652, 2018.

[30] D. A. Vargas-Inchaustegui, A. E. Hogg, G. Tulliano et al., "CXCL10 production by human monocytes in response to Leishmania braziliensis infection," Infection and Immunity, vol. 78, no. 1, pp. 301-308, 2010.

[31] V. Iniesta, L. Carlos Gómez-Nieto, I. Molano et al., “Arginase I induction in macrophages, triggered by Th2-type cytokines, supports the growth of intracellular Leishmania parasites," Parasite Immunology, vol. 24, no. 3, pp. 113-118, 2002.

[32] T. M. Cardoso, Á. Machado, D. L. Costa et al., "Protective and pathological functions of $\mathrm{CD}^{+} \mathrm{T}$ cells in Leishmania braziliensis infection," Infection and Immunity, vol. 83, no. 3, pp. 898-906, 2015.

[33] D. Santos, T. M. Campos, M. Saldanha et al., "IL- $1 \beta$ production by intermediate monocytes is associated with immunopathology in cutaneous leishmaniasis," Journal of Investigative Dermatology, vol. 138, no. 5, pp. 1107-1115, 2018.

[34] T. M. Campos, F. O. Novais, M. Saldanha et al., "Granzyme B produced by natural killer cells enhances inflammatory response and contributes to the immunopathology of cutaneous leishmaniasis," The Journal of Infectious Diseases, vol. 221, no. 6, pp. 973-982, 2020.

[35] F. O. Novais, B. T. Nguyen, D. P. Beiting et al., "Human classical monocytes control the intracellular stage of Leishmania braziliensis by reactive oxygen species," The Journal of Infectious Diseases, vol. 209, no. 8, pp. 1288-1296, 2014.

[36] D. S. Lima-Junior, D. L. Costa, V. Carregaro et al., "Inflammasome-derived IL- $1 \beta$ production induces nitric oxide-mediated resistance to Leishmania," Nature Medicine, vol. 19, no. 7, pp. 909-915, 2013.

[37] P. P. Carneiro, J. Conceição, M. Macedo, V. Magalhães, E. M. Carvalho, and O. Bacellar, "The role of nitric oxide and reactive oxygen species in the killing of Leishmania braziliensis by monocytes from patients with cutaneous leishmaniasis," PLoS One, vol. 11, no. 2, article e014808, 2016. 